FERMILAB-Conf-96/436

\title{
BTEV - A Dedicated $B$ Physics Detector at the Fermilab Tevatron Collider
}

\author{
J.N. Butler \\ Fermi National Accelerator Laboratory \\ P.O. Box 500, Batavia, Illinois 60510
}

November 1996

Proceedings of the 2nd International Conference on Hyperons, Charm and Beauty Hadrons, Montreal, Quebec, Canada, August 27-30, 1996. 


\section{Disclaimer}

This report was prepared as an account of work sponsored by an agency of the United States Government. Neither the L'nited States Government nor any agency thereof, nor any of their employees, makes any warranty, express or implied, or assumes any legal liability or responsibility for the accuracy, completeness or usefulness of any information, apparatus, product or process disclosed. or represents that its use would not infringe privately owned rights. Reference herein to any specific commercial product, process or senvice by trade name, trademark, manufacturer or otherwise, does not necessarily constitute or imply its endorsement, recommendation or favoring by the United States Government or any agency thereof. The views and opinions of authors expressed herein do not necessarily state or reflect those of the United States Government or any agency thereof.

\section{Distribution}

Approved for public release: further dissemination unlimited. 


\title{
BTEV - A Dedicated $B$ Physics Detector at the Fermilab Tevatron Collider
}

\author{
J. N. Butler ${ }^{\mathbf{a}}$

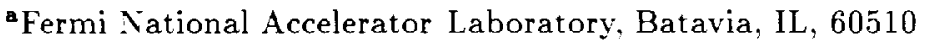 \\ The capabilities of future Dedicated Hadron Collider $B$ Physics experiments are discussed and compared to \\ experiments that will run in the next few years. The design for such an experiment at the Tevatron Collider is \\ presented and an evolutionary path for developing it is outlined.
}

In this paper, we describe the BTEV experiment at the Fermilab Collider - a Dedicated $B$ Physics Experiment whose acceptance is in the forward direction. In the first section, we discuss the role of dedicated experiments at hadron colliders in the the study of $B$ decays and compare their goals with those of other experiments. In the second section, we present the key features of the BTEV experiment. Section three presents the results of the some of the simulation studies that have been carried out to date for the BTEV design. We conclude by presenting an evolutionary path for developing and constructing the BTEV experiment at the Fermilab Tevatron.

\section{The Role of Dedicated Experiments at Hadron Colliders in the the Study of $B$ Decays}

$B$ physics is an extremely interesting and exciting subject for investigation because fundamental parameters of the Standard Model can be measured and physics beyond the Standard Model might be observed. We believe that a Dedicated Hadron Collider $B$ Physics Experiment is an essential follow-on to the planned asymmetric electron-positron collider experiments, the symmetric electron-positron collider experiment, and other 'first generation' hadron CP experiments.

Evolution of knowledge on $B$ Decays has developed slowly over the period of almost 20 years since the $\Upsilon$ [1] was discovered. Its past, present, and future development may be viewed as going through at least 4 phases [2]:
1. Initial discovery and characterization as a weakly decaying quark. This includes the period from the discovery of the $\Upsilon$ (FNAL), to the observation of the narrow $\Upsilon$ states at DORIS, the discovery of the the $\Upsilon(4 S)$ at CLEO, and early indications of the long $B$ lifetime;

2. Detailed characterization of properties and decays. This includes reconstruction of many $B$ decay modes (especially those containing $D$ 's and $\psi$ 's) by ARGLS and CLEO, the detailed study of semileptonic decays, and the observation of mixing. At LEP and at Fermilab, the $B_{a}$ meson and $\Lambda_{b}$ were observed, $B$ lifetimes were measured, evidence was found for $B^{* *}$ 's and higher mass $B$-baryons, and the $B^{\circ}$ mixing parameter was obtained from time-dependnent studies. CLEO's observations of 'rare decays' marks the transition to the next phase;

3. Rare Decays and decay asymmetries. The observation by CLEO of penguins: $B \rightarrow$ $s \gamma$ and $B \rightarrow K^{*} \gamma$; of suppressed two body modes: $B^{\circ} \rightarrow \pi^{+} \pi^{-}, K^{+} \pi^{-}$and of exclusive charmless semileptonic decays is the beginning of this phase. I BELIEVE THIS IS OLR CLRRENT STATLS. Ahead is the measurement of more rare decays, more precise measurement of $\pi^{+} \pi^{-}$and $K^{+} \pi^{-}$, and the first measurements of some CP asymmetries - almost certainly those related to $\sin 2 \beta$, possibly other angles of the unitarity triangle, and possibly some 
direct $\mathrm{CP}$ violating modes. The experiments which are going to be running and hopefully will accomplish the remainder of the work of this phase are: PEPII/BaBar, KEK/Belle, CESR/CLEO, CDF/D0, and HERA-B. While many claims are made that these experiments will answer all remaining questions in $B$ physics, there is a school of thought that says that their accomplishments, while great, will still not exhaust the field of investigation. It is almost certain that even after the successes of these experiments one will still want to go on to a fourth phase of $B$ physics;

4. Detailed measurements of decay asymmetries, in particular of those giving the angle $\gamma, B_{a}$ mixing, studies of other $B$ hadrons, and very sensitive searches for rare and Standard-Model-forbidden decays.

The $e^{+} e^{-}$experiments offer a very clean environment in which to study $B$ physics. However, even at design luminosity on the $\Upsilon(4 S)$, the total number of produced $B$ 's is only about $3 \times 10^{7}$ per year. This is adequate, given the quality of the detectors, to make a first measurement of $\sin 2 \beta$, once the design luminosity is approached. Many other goals, such as precision measurements of $\alpha$ and $\gamma$ and measurements of $x_{a}$, are unlikely to be achieved based on our calculations. In fact, it is hard to see the experiments even running on the $\Upsilon(5 S)$ in order to begin to look at $B$, states until several years into their lifetime and even then the experimental challenges are such that good measurements are not likely. Since the detectors and triggers are already designed to be highly efficient, the only way to improve the situation is to achieve increases in machine luminosity. 'There is no clear path to an increase of even a factor of three at present.

Hadron experiments currently in the works, such as CDF and HERA-B will aim at roughly the same level of sensitivity as the $e^{+} e^{-}$experiments. HERA-B suffers from a low cross section. CDF suffers from low efficiency due to the soft momentum spectra of the produced $B$ 's and severe triggering problems. Estimates suggest sensitivity to $\sin 2 \beta$ and (much) more optimistically to $\sin 2 \alpha$.
These kind of estimates have been wrong before because they have not been robust against the assumptions that go into them.

We think that it is likely that some of these experiments will see evidence for $C P$ violation in $\psi K$, but are convinced that they will not have anywhere near the precision to do a comprehensive set of precision measurements. Each experiment faces fundamental limitations which make it hard to push it too far beyond the sensitivity achieved in the first few years of running. They will, however, also learn many new facts about $B$ physics which will help in the interpretation of future results. Some of these studies are best carried out in an $e^{+} e^{-}$machine because they require reconstruction of neutrals which is made easier in the relatively clean environment.

To quote a colleague, 'This round of experiments is likely to open the study of $\mathrm{CP}$ violation in $\mathrm{B}$ decays but it is unlikely to close it.'

The fourth phase will, therefore, need to be carried out at high energy hadron collliders which are the only machines that produce enough $B$ 's to advance beyond the generation of experiments which are now being constructed. The next generation of hadron colliders, the Tevatron after the Main Injector upgrade and the LHC, will certainly have adequate luminosities. The challenge is to design a detector that can be efficient for detecting and tagging $B$ decays in the hostile (rate, radiation damage) and complex ( high multiplicities, many background events, extra jets even in $B$ events) environment in these machines.

\section{The BTEV experiment at the Fermilab Tevatron Collider}

The challenge for any future hadron collider B experiment is:

- To design an experiment that achieves high efficiency in the hostile and complex environment of a hadron collider. This requires the effective and aggressive use of the most modern technologies.

- To represent a significant step beyond what will be achieved by the upcoming generation of experiments and their possible ex- 
tensions and upgrades.

- To be competitive with other hadron collider $B$ experiments which could be taking data in the same time frame. In the case of BTEV, the competition is LHC-B.

Some parameters of the Tevatron that must be taken into account in creating a design to meet these challenges are given in table 1.

Table 1

A Few Tevatron Parameters Relevant to BTEV

\begin{tabular}{|l|l|}
\hline Property & Value \\
\hline Luminosity & $2 \times 10^{32}$ \\
Bunch Spacing & $132 \mathrm{~ns}$ \\
Length of Luminous Region & $\sigma_{z}=30 \mathrm{~cm}$ \\
Transverse X dimensions & $\sigma_{x}=30 \mu \mathrm{m}$ \\
Transverse Y dimensions & $\sigma_{y}=30 \mu \mathrm{m}$ \\
Interactions/crossing & mean $=2$ \\
B Cross Section & $50 \mu$ barn \\
$\sigma_{b b} / \sigma_{\text {inel }}$ & $\sim 10^{-3}$ \\
\hline
\end{tabular}

Over the last year, BTEV has begun to take shape based on an understanding of what needs to be done to meet these challenges. A reference design now exists. These are the key design features of BTEV:

- a dipole located on the IR, which gives BTEV an effective 'two arm' acceptance;

- a precision vertex detector based on planar pixel arrays;

- a vertex trigger at Level I which makes BTEV very efficient for states even if they have no leptons in them; and

- strong particle identification. Especially important is the requirement of very good charged hadron identification. Many of the states that will be of interest in this phase of $B$ physics will only be separable from other states if this capability exists. Also, it will allow for the possibility of kaon tagging. Muon and electron identification are also important for tagging and for studies of decay modes involving leptons.
The motivation for a vertex trigger is explained very well in the LHC-B Letter of Intent [3]:

"At LHC energies, $B-\bar{B}$ events look very much like minimum-bias events, apart from having detached secondary and possibly tertiary vertices and a somewhat higher transverse momentum for the $B$-hadron decay products."

The tracking system has to be designed together with the trigger electronics to realize the goal of a Level I vertexing trigger.

A schematic of the experiment is given in figure 1. We now discuss the key elements of the reference design.

\subsection{Dipole centered on IR - a.k.a. 'Cen- tral Dipole'}

The original motivation for this was to increase the acceptance. It was also realized that the extended IR and the limited space in $z$ in the available collider halls at Fermilab made it difficult to make a geometry for a single arm that preserved the acceptance and did not produce a strong and oddly shaped field in at least part of the collision region. The central dipole arrangement gives us acceptance from $10 \mathrm{mr}$ to $300 \mathrm{mr}$ in both hemispheres. It may be viewed as the limiting case of two arms being pushed closer and closer to the center of the IR until they merge.

It was soon understood that having a large uniform field at the vertex detector was a virtue for the trigger since it permits a crude momentum measurement using only the elements of the vertex detector and this information will be available to the first level trigger. This allows one to eliminate tracks whose resolution is dominated by multiple Coulomb scattering.

The dipole in the reference design has a central field of 15 kGauss. The pole face can widen beyond the IR but the overall integrated field strength needs to be ahout 75 KGauss-meters. The overall length is around $6 \mathrm{~m}$ and the aperture at the downstream end is around $\pm 1 \mathrm{~m} \times \pm 1 \mathrm{~m}$.

\subsection{Tracking System}

In designing a vertex tracking system, one must consider the long interaction region of the Tevatron which has a $\sigma_{\boldsymbol{z}}$ of $30 \mathrm{~cm}$. This forces one to have a rather long vertex detector. On the 


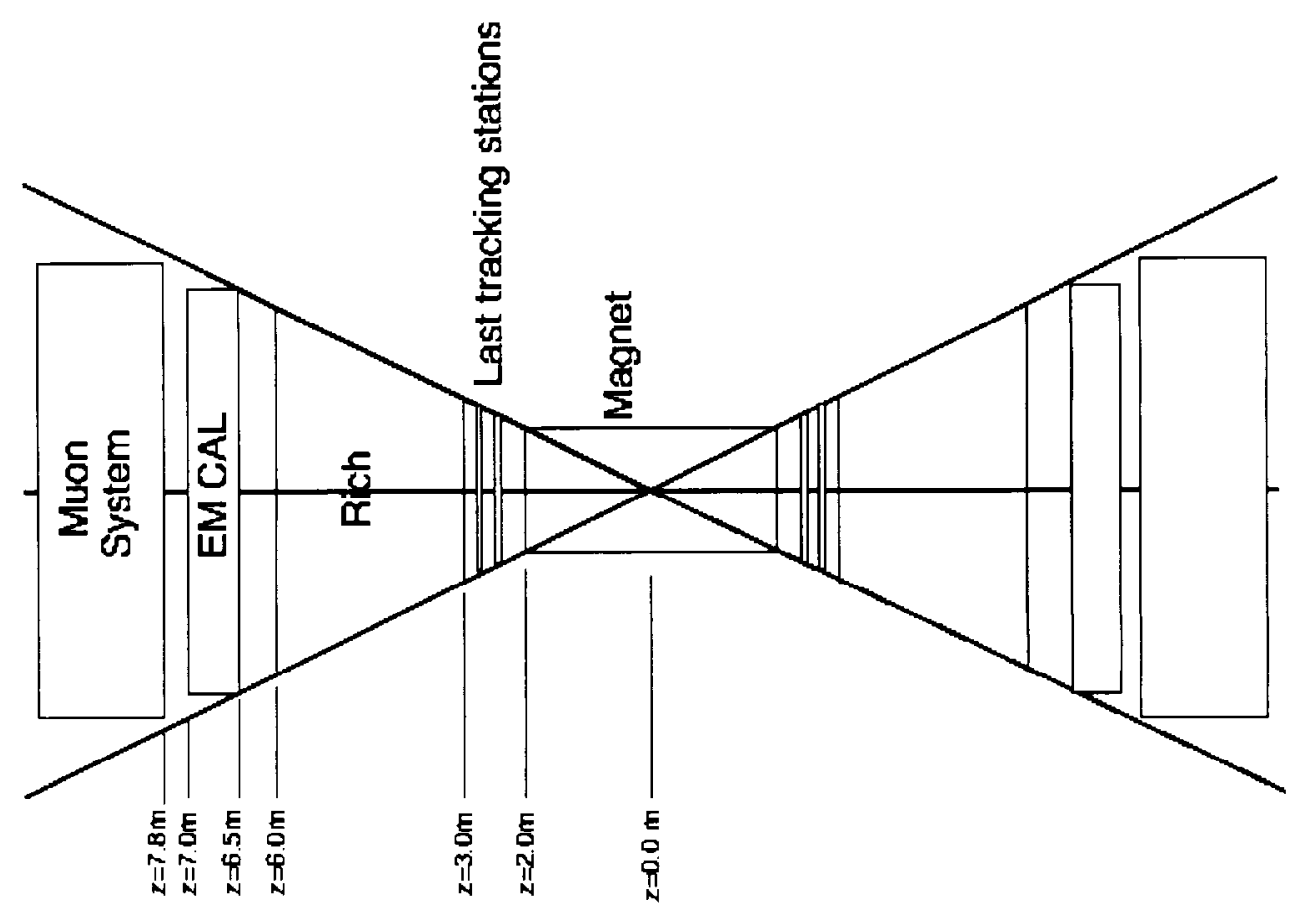

Figure 1. Schematic representation of the BTEV detector

other hand, it is actually a positive feature which partially compensates for the long bunch spacing. It may well be possible to work at luminosities higher than the design point of $2 \times 10^{32}$ because each little section of $z$ is effectively monitored by its own 'quasi-independent' set of detector planes.

The 'reference tracker', shown in figure 2 has triplets of planes arrayed along the IR separated by about $4 \mathrm{~cm}$. Each triplet consists of one wafer that measures the bend view, one that measures the non-bend view, and a third that measures the bend view again. The pixels are $30 \mu$ by $300 \mu$. The pulse height is read out and made available to the trigger so that one can actually derive a crude momentum measurement over three or four triplets. This can be used to reject very soft tracks, whose mulitple scattering would make the information from this track confusing to the trigger algorithm.

The implementation of this system will be very difficult. Because pixel information must be made available to the trigger, a custom electronic design is necessary. Radiation damage is an issue for the inner edge of the detector where the fluence can reach values of $2 \times 10^{14}$ per $\mathrm{cm}^{2}$ per year. There are also significant mechanical and thermal issues that must be addressed.

\subsection{Triggering System}

BTEV's trigger focuses on the key difference the presence of detached vertices - at Level I. It is very efficient for the $B$ decays that can be successfully reconstructed by the spectrometer and used for analysis. It also avoids the need to focus on specific final states at Level I which can limit the accessible physics.

Use of pixels, with pulse height, for the tracker will produce very high spatial resolution for the trigger and the final analysis. If pixels with pulse height achieve the expected resolution of around 6 microns, this provides real momentum information, clean pattern recognition, and precision 


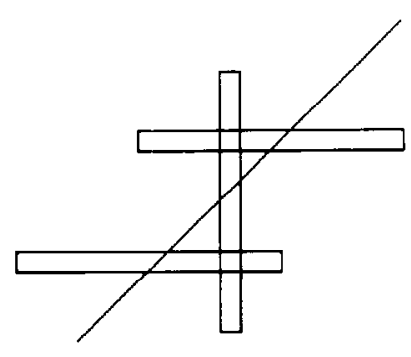

\section{Detauls of Reterence P1xel Detector}

\section{Pixel Orientation in Triplet}

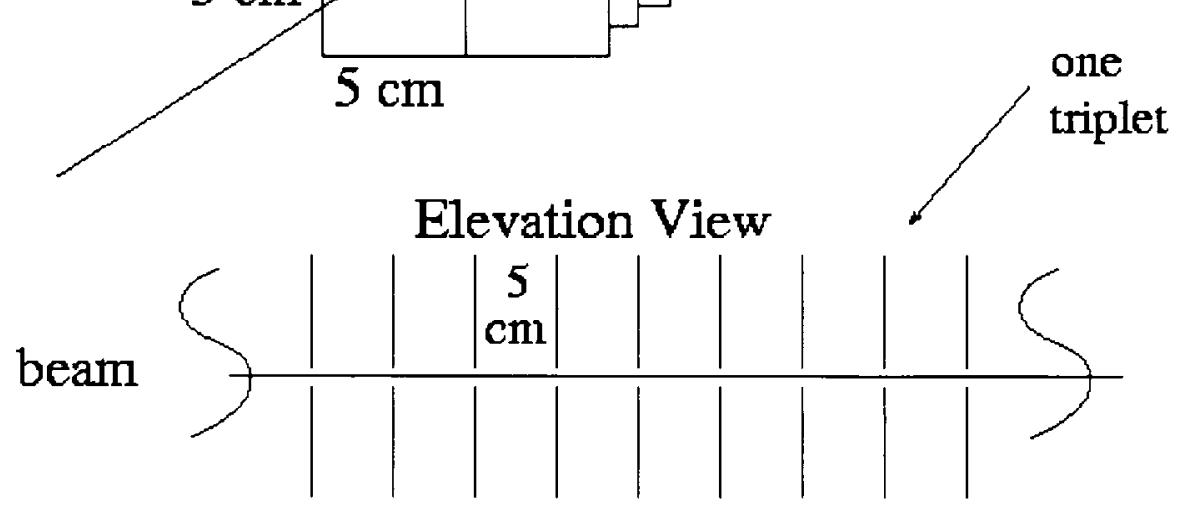

Figure 2. 'Reference' pixel tracker

track extrapolations to the level I trigger.

Deriving the vertex trigger from the pixels without matching to the downstream (coarser and slower) elements provides very high speed tracking and triggering and makes it possible to operate at very high luminosities. In the trigger, BTEV relies on fast, low occupancy elements (the silicon vertex detector) which are relatively immune to pileup and random overlaps.

The reference trigger scheme we show was dcveloped by the Penn Group - Selove, Sterner, and Izik [4]. While it may undergo some revisions, we are sufficiently encouraged by the initial results that all our simulation and hardware efforts currently are directed towards this approach.

The basic idea of the trigger is to divide the vertex detector into $\phi$ slices each of which has an occupancy that is fairly small - typically about one half track per slice per event - and to consider each an independent tracking problem. The reference design uses $32 \phi$ slices. Pixel information from each $\phi$ slice is preprocessed by 'hit processor' electronics and the digitized hits - which are really minivectors - are sent via a switching network to a farm of processors. In the current design, there are 64 processors per $\phi$ slice or 2048 total processors.

The processors receive 'minivectors' from each triplet consisting of an $x, y, z$ coordinate and a slope $\frac{d y}{d x}$. The hits are sorted into two sets depending on the sign of $\frac{d y}{d z}$. These are also ordered according to $\mathrm{z}$. This produces two $\mathrm{z}$-ordered lists: one of 'forward' going tracks and one of 'backward' going tracks.

The bendview hits are processed as followed:

- A track is traced from the upstream end in 
z.

- The algorithm takes the average of the $\frac{d y}{d z}$ in the current plane with the $\frac{d y}{d z}$ in the next plane. It uses this average slope (which represents the slope of the chord of the magnetically deflected trajectory) to project from the first plane into the second plane.Then, it checks to see whether the $y$-value of the minivector in the downstream plane agrees with the projection.

- This process is continued until no link is found in a $z$ plane.

- If three or more hits satisfy the tracking requirements, the string of minivector coordinates are fed to a fitting stage which uses a fast fitting algorithm to get the momentum of the track candidate.

- The resulting track with its measured momentum is then traced backwards in both $\mathrm{x}$ and $y$ to the interaction region to get an impact parameter (this assumes that the beam $\sigma$ is small compared to the expected impact parameter from $B$ decays. If it is not, then a vertex algorithm would be used). An impact parameter and quality factor (approximate normalized error) are obtained.

The way in which this track information is used to form the Level I trigger is described below in the section on simulations.

A design for implementing such an algorithm in hardware has been worked out. Prototype boards are being developed. Estimates of the time to carrying out the tracking algorithm are in the neighborhood of $20 \mu \mathrm{s}$.

The Level I trigger we plan must be capable of reducing the trigger rate to a few khiz. We plan to take all the data from vertex detector at the full crossing rate of $10 \mathrm{MHz}$ off the detector. This amounts to $100 \mathrm{Gbytes} / \mathrm{s}$. The track information goes to a bank of vertex processors which operate on the set of individual tracks from each interaction. The event rate is then reduced down to something like $50 \mathrm{kHZ}$ if the only test made is for a detached vertex in vacuum. The Level I $\rightarrow$
Level II bandwidth is sufficient to transfer all this data. However, the data rate out of Level I can be much lower if we also make tests on the effective mass at the detached vertex. Since most of the detached-vertex events will be $K_{s}$ or charm. a mass cut can reduce the rate to a few $\mathrm{kHz}$ with almost no bias against $B$ events of interest. Only when we get to this ratc do we have to read out the entire detector.

\subsection{Particle Identification:}

Only a limited amount of work has been done on particle identification so far but it is a crucial part of the experiment. The momentum range that needs to be covered is large, extending from $\sim 1$ to $70 \mathrm{GeV} / \mathrm{c}$. This is determined by studying the momentum spectra of particles in a variety of $B$-meson final states, including $B \rightarrow \pi^{+} \pi^{-}$ and $B_{s} \rightarrow K^{*} \psi$, which is a proposed mode for measuring $B$, mixing [5], [6].

The reference design uses two Ring Imaging Cerenkov counters to cover this large momentum range:

1. A sawtooth LiF radiator (thickness about 1 cirl) followed by a $20 \mathrm{~cm}$ expansion gap and a proximity focussed photon detector. This detector provides good $\pi-\mathrm{k}$ spearation from $0.5 \mathrm{GeV} / \mathrm{c}$ to $3.5-4.0 \mathrm{GeV} / \mathrm{c}$.

2. A $C_{2} F_{6}$ gas radiator of length $2.5 \mathrm{~m}$ with a system of focusing mirrors. The plan is to use TEA as the photosensitive material. This counter covers the momentum range from around $3.5 \mathrm{GeV} / \mathrm{c}$ to about $70 \mathrm{GeV} / \mathrm{c}$.

Other options are being considered.

\section{Simulation Studies}

The fourth generation $B$ experiment must go beyond the measurement of $\mathrm{CP}$ asymmetry in $B_{d} \rightarrow \psi K_{d}$ and must do a very complete program of rare $B$ physics. It therefore needs to have good performance for a wide range of final states such as: $B^{\circ} \rightarrow \pi^{+} \pi^{-}, B \rightarrow D^{\circ} K$ and $B \rightarrow D_{c p}^{\circ} K, B^{\circ} \rightarrow D^{+} D^{-}$and $D^{*+} D^{*-}$, various $B$, mixing modes including $\psi K^{* 0}$, a variety of $B$ baryon modes, $B_{c}$ modes, rare decays, and many others. To insure this, simulation studies 
are being carried out with the simulation package MCFAST [7].

One study which has been done is $B \rightarrow \pi^{+} \pi^{-}$. The interest first is to establish the fact that this design can achieve adequate efficiency and background rejection to measure $\sin 2 \alpha$. The main background to this state was found to come mainly from 'generic' $B \bar{B}$ events which are more than $10^{5}$ times more numerous (rather than from minimum bias events or charm events).

We have done a detailed analysis using 40,000 $B \rightarrow \pi^{+} \pi^{-}$events and $300,000 B \bar{B}$ events. For this study, we actually used a single arm forward spectrometer. This work has been reported elsewhere [8]. We are in the process of running a much larger sample using the reference central dipole geometry. The yield is almost exactly a factor of two higher as expected. When this is complete, we will have an accurate measurement of the signal-to-background in this very demanding final state.

\subsection{Trigger simulations of $B \rightarrow \pi^{+} \pi^{-}, B \rightarrow$ $\psi K^{*}$, and charm}

The trigger is based on calculation at level I of the impact parameter of tracks relative to a reconstructed 'seed' vertex. For each track, a crude momentum and an impact parameter are calculated. A cut on $P_{y}$ is placed on all tracks used in the trigger. The value of the $P_{y}$ cut is between $0.25 \mathrm{GeV} / \mathrm{c}$ and $0.75 \mathrm{GeV} / \mathrm{c}$. A primary vertex requires at least three tracks to intersect in the beam region. The trigger is then determined by requiring a certain number of tracks, $N$, that were not in the primary vertex to have impact parameters greater than some cut, $b_{\min }$. Preliminary results for the efficiencies of the trigger for light quark and charm background, and two $B$ signals for various values of $N$ and $b_{\min }$ are shown in figure 3 .

\section{An evolutionary path to designing and building BTEV}

Pecently, FNAL has been considering a plan to open up a new colliding beam area for experiments at the location on the ring known as $\mathrm{C} 0$. One strong motivation is to provide a place where ideas for future technology development, including those required for heavy quark experiments, can be carried out. It is also possible that small scale prototypes of future detectors can begin to do actual experiments in this area to validate the integrated experiment design.

\subsection{Need for $R \& D$}

While advances in technology have pointed the way to meeting all the triggering, event building, and DA challenges, much R\&D is required to demonstrate workable solutions and to optimize them. In addition, pixel $\mathrm{R} \& \mathrm{D}$, including electronics design and simulation, is necessary for the tracking system. This work includes prototype design, prototype fabrication, and testing. FNAL is beginning to organize an effort, almost surely in collaboration with other U.S. groups that have been working in this area.

\subsection{Co tests - preliminary thoughts}

With lab support, we are now designing a rigorous test of our triggering and tracking concepts to run in the $\mathrm{CO}$ interaction region during the first Inain injector run [9]. The BTEV collaboration is excited by this possibility and sees it as an excellent way to develop the techniques necessary for this challenging experiment. The initial effort will be directed at a test of the tracking and trigger concepts in the collider environment by making simple measurements of vertex resolution.

While we accept the fact that resources are scarce for $R \& D$ efforts, we have suggested that the lab view all of requests for support in the next few years in the context of preparing for the $\mathrm{C} 0$ tests and eventually for the full BTEV experiment. In particular, we have urged the lab to initiate a program of pixel $R \& D$ which has as one of its goals the development of a pixel detector suitable to the triggering and tracking requirements of BTEV.

We have been asked if there are any physics demonstrations that could be used to prove the soundness of our approach that could be undertaken in $\mathrm{C} 0$ with limited resources and a luminosity of $10^{31}$. We have come up with two ideas:

- A charm experiment. Per month of run- 
ning, one can get about $10^{7}$ fully reconstructed $K \pi$ or $K \pi \pi$ decays! A particle identifier would be essential. A downstream tracker on one side of the dipole is required. A muon detector could be used as an independent trigger which would then enable us to make a high statistics absolute calibration of the vertex trigger's efficiency on $D^{o}$ 's and $D^{+}$'s. We are investigating the changes we would have to make to our triggering scheme to make it more efficient for charm.

- An experiment to observe and study $B^{\circ} \rightarrow$ $\psi K_{s}, B_{s}$ mixing, and $B$-baryons. A downstream tracker and muon detector is required. A serious attempt to detect $B$ baryons probably needs a reasonably good particle identification system.

Charm is not only a possible test but also an important secondary goal of the complete BTEV experiment.

\section{Conclusions}

While there is obviously a long way to go before one could even claim to have a complete conceptual design, the BTEV experiment is beginning to take shape. Some major design decisions have been made. Preliminary design efforts have not encountered any 'show stoppers' and work continues. Fermilab's proposed new initiative to establish a collision region at $\mathrm{C} 0$, which can serve (among other purposes) as a home for testing the trigger and tracking components of BTEV, is a great breakthrough. For the first time, we see a real path for developing the experiment so that a proof of principle can be accomplished by 2000 2001. At that time, a full technical proposal will be available and the lab would have to make a final decision to proceed and a final decision on the siting of the experiment. With much of the system, the tracking and trigger components, alrcady partially implemented, the experiment can be constructed, installed, and debugged in stages and ready for a full implementation around the year $2004-2005$. It is possible to imagine staging a sequence of tests, some of which would consti- tute physics measurements, and which taken together would teach us how to do the experiment well before the final apparatus was complete.

\section{Acknowledgements}

I would like to thank my colleagues in the Dedicated $B$ Collider Group and the Fermilab Simulation

Group for their help in preparing this paper and the conference organizers for conducting an excellent conference. This work was supported by the Fermi National Accelerator Laboratory, which is operated by Universities Research Association, Inc., under contract DE-AC02-76CH03000 with the U.S. Department of Energy.

\section{REFERENCES}

1. S.W. Herb et al. (CFS Collaboration), Phys. Rev. Lett. 39(1977) 252.

2. For a good historical overview, see $\mathrm{A}$. $\mathrm{Ali}$, " $B$ Decays- Introduction and Overview", in " $B$ Decays, revised second edition", edited by Sheldon Stone, World Scientific Press, Singapore(1994). For reviews of recent results on $B$ lifetimes, excited $B$ mesons, and $B$ mixing, see these proceedings. For a summary of recent results on rare $B$ decays from CLEO, see Sheldon Stone's article:

3. LHC-B Letter-of-Intent, A Dedicated LHC Collider Beauty Experiment for Precision Measurements of CP Violation, CERN/LHCC 95-5 (25 August 1995)

4. R. Izik et al., " Preliminary Monte Carlo Results for a Secondary-Vertex Trigger with Online Tracking", UPR-234E and D. Husby, P. Chew, K. Sterner, and W. Selove, Design of a Secondary Vertex Trigger System for a Hadron Collider.

5. $B$, Mixing via $\psi \bar{K}^{*}$, P.M. McBride and S. Stone, Nulcear Instruments and Methods in Physics Research, A368 (1995), p38-41.

6. McBride, P.M., A Dedicated B Experiment at Fermilab, to appear in the proceedings of Beauty96, Nulcear Instruments and Methods in Physics Research

7. The MCFAST Detector Simulation Package, http://fnpspa.fnal.gov/mcfast.html

8. $B$ Physics at the Fermilab Tevatron after the Year 2000, J.N. Butler, Nulcear Instruments and Methods in Physics Research, A368 (1995), p145-151.

9. BTEV Expression of Interest in $\mathrm{CO}$, A. Santoro et al., submitted to Fermilab Program Planning Office, June 5, 1996. 

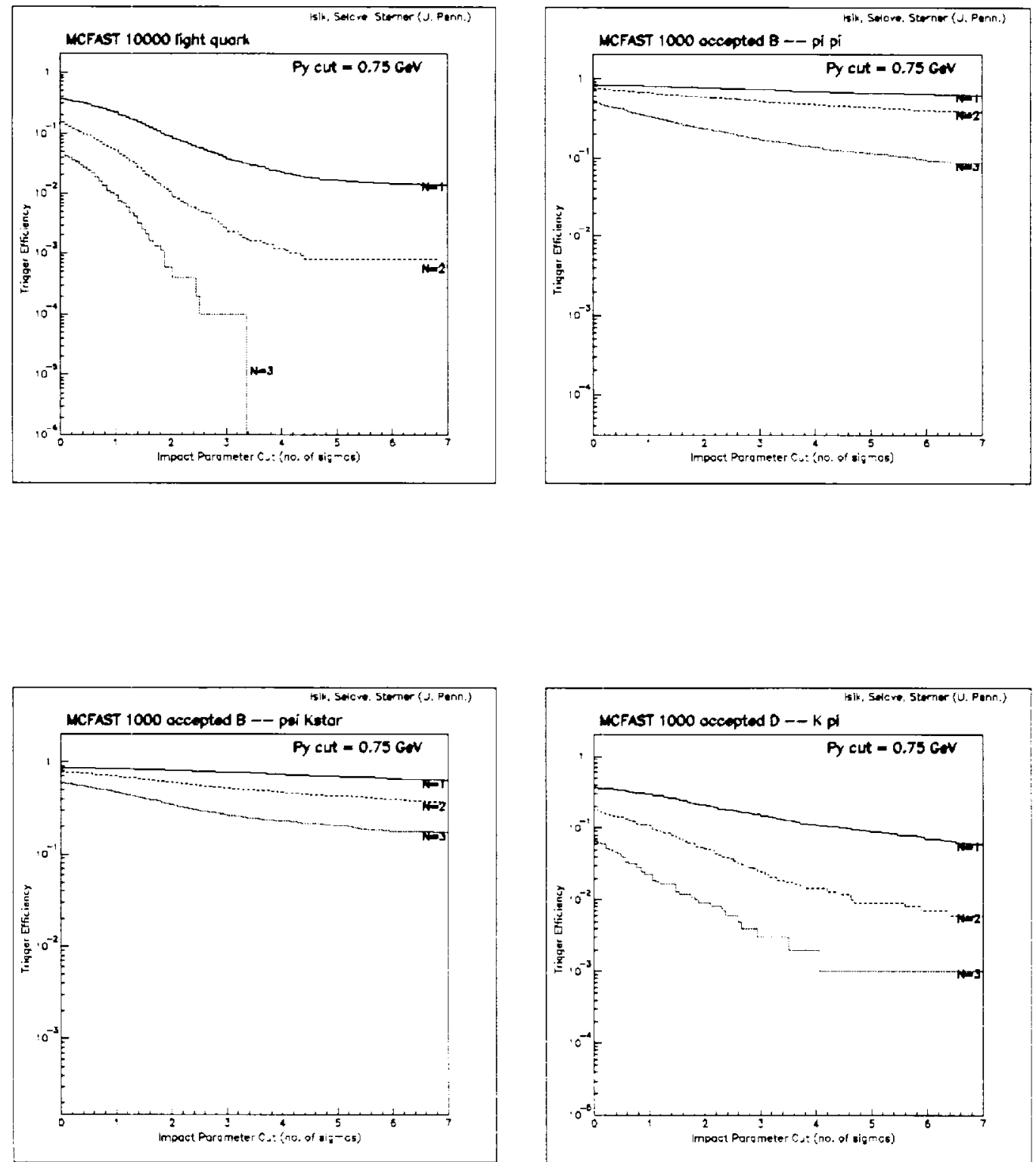

Figure 3. upper left) Trigger efficiency for light quark events; upper right) Trigger efficiency for $B^{\circ} \rightarrow$ $\pi^{+} \pi^{-}$; lower left) Trigger efficiency for $B^{\circ} \rightarrow \psi K^{*}$; and lower right) Trigger efficiency for charm ( $D^{\circ} \rightarrow$ $\left.K^{-} \pi^{+}\right)$. 